\title{
Evaluation of Nutritional Habits and Dietary Management in Children with Chronic Kidney Disease
}

\author{
Kronik Böbrek Hastalığı Olan Çocuklarda Beslenme Alışkanlıklarının \\ ve Diyet Yönetiminin Değerlendirilmesi
}

Nevra KOÇ ${ }^{1}$, Hülya YARDIMCl ${ }^{2}$, Nazıı Nur ASLAN², Umut Selda BAYRAKÇI ${ }^{3}$

\author{
'University of Health Sciences, Ankara Child Health and Diseases, Hematology Oncology, Training and Research Hospital, \\ Department of Nutrition and Dietetics, Ankara, Turkey \\ ${ }^{2}$ Ankara University, Faculty of Health Sciences, Department of Nutrition and Dietetics, Ankara, Turkey \\ ${ }^{3}$ Yıldırım Beyazit University, Medical School of Faculty, Department of Pediatric Nephrology, Ankara, Turkey
}

\begin{abstract}
Objective: This study was conducted to evaluate the dietary habits, nutritional status and dietary management practices of children with chronic kidney disease.

Material and Methods: The study was conducted on 16 patients aged 2 to 18 years ( 9 boys, 7 girls) who were followed at our clinic with predialysis chronic kidney disease and with peritoneal dialysis. The nutritional status of the patients was determined by 24-hour dietary recall and subjective global nutritional assessment. In addition, anthropometric measurements and biochemical parameters were evaluated.

Results: When the anthropometric measurements of the children were evaluated; $56.2 \%$ of them were found to be wasted and $62.5 \%$ were found to be stunted. According to subjective global nutritional assessment, $60 \%$ were found to be severely malnourished. Iron binding capacity, glomerular filtration rate and albumin levels were significantly higher in the predialysis group than in the peritoneal dialysis group $(p<0.05)$. When uric acid, total protein, hemoglobin, hematocrit, ferritin, iron, sodium, potassium, and phosphorus levels were examined, no difference was found between the groups ( $p>0.05$ ) The average daily energy intake of the children was $1564.3 \pm 982.4 \mathrm{kcal}$. Carbohydrates were found to be the source of $45.6 \pm 9.0 \%$ of the daily energy intake while $12.4 \pm 4.1 \%$ of the energy intake was from proteins and $41.9 \pm 7 \%$ from fats. We found that children with chronic kidney disease had consumed dietary fibers, calcium and magnesium inadequately while consuming salt more than their requirements.
\end{abstract}

Conclusion: Children with chronic kidney disease had irregular eating habits and their dietary management was inadequate. Attitudes, behaviors and knowledge of these children and their families were inadequate regarding the patients's dietary needs. For this reason, it is believed that a diet based on individual nutrition rather than a nutrient-based dietary approach consisting of a chain of restrictions is more suitable for children with chronic kidney disease.

Key Words: Children, Chronic kidney disease, Dietary habits, Dietary management, Nutritional assessment

\section{Öz}

Amaç: Çalışma kronik böbrek hastalığı olan olan çocukların beslenme alışkanlıklarını, malnütrisyon durumlarıı ve diyet yönetimlerindeki uygulamalarıı değerlendirmek amacıyla yapıımışıı.

Gereç ve Yöntemler: Kliniğimizde izlenen ayaktan ve yatarak tedavi gören prediyaliz ve periton diyalizi uygulanan kronik böbrek hastalı̆ı olan 2-18 yaşları arasında 16 (9 erkek, 7 kı) gönüllü hasta çalışmaya dahil edilmiştir. Hastaların beslenme durumu, 24 saatlik geriye dönük besin tüketim kaydı ve subjektif global nutrisyonel değerlendirme yöntemi ile belirlenmiştir. Ayrıca antropometrik ölçümleri, biyokimyasal parametreleri değerlendirilmiştir.

Bulgular: Hastalar antropometrik ölçümlerine göre değerlendirildiğinde; \%56.2'sinin kavruk, \%62.5'inin bodur olduğu bulunmuştur. Subjektif Global Nutrisyonel Değerlendirme yöntemine göre \%60.0'ı ağır malnütrisyonludur. Prediyaliz grubunda periton diyalizi grubuna göre demir bağlama kapasitesi, glomerül filtrasyon hızı ve albümin seviyesi anlamlı olarak daha yüksek bulunmuștur ( $p<0.05)$. Ürik asit, total protein, hemoglobin, hematokrit, ferritin, demir, sodyum, potasyum, fosfor seviyeleri incelendiğinde, gruplar arasında fark bulunmamışıı ( $p>0.05$ ). Çocukların günlük enerji alımı ortalama $1564.3 \pm 982.4$ kcal'dir. Alınan enerjinin \%45.6 \pm 9.0 'u karbonhidrattan, \%12.4 \pm 4.1 'i proteinden, \%41.9 \pm 7.0 'si yağdan gelmektedir. Hastaların diyet posasını, kalsiyum ve magnezyumu yetersiz aldıkları, tuz tüketiminin ise gereksinimden fazla olduğu saptanmıştır.

Correspondence Address / Yazışma Adresi:

Nevra KOÇ

University of Health Sciences, Ankara Child Health and Diseases,

Hematology-Oncology Training and Research Hospital, Ankara, Turkey

E-mail: nevrakoc@yahoo.com
Received / Geliş tarihi : :23.02.2018 Accepted / Kabul tarihi : 20.04.2018

Online published 01.06 .2018

Elektronik yayın tarihi

DOI: 10.12956/tjpd.2018.365 
Sonuç: Çalışmaya alınan kronik böbrek hastalığı olan çocukların düzensiz beslenme alışkanlıkları mevcut olup, diyet yönetimleri oldukça zayıftır. Ailelerinin ve kendilerinin beslenme konusundaki eğitimleri, tutum ve davranışları yetersizdir. Bu nedenle kronik böbrek hastalığı olan çocuk hastalarda yasaklar zincirinden oluşan besin ögesi odaklı bir diyet yaklaşımı yerine bireysel beslenme odakı bir diyet yaklaşımının daha doğru olduğu düşünülmektedir.

Anahtar Sözcükler: Çocuklar, Kronik böbrek hastalığı, Beslenme alıșkanlıkları, Diyet yönetimi, Beslenme durumunun değerlendirilmesi

\section{INTRODUCTION}

In the chronic kidney diseases (CKD), nutritional status is the most important factor that may influence the growth rate, development of brain and other organs, and protection of muscles and fat tissue (1).

Malnutrition is the most common clinical picture in children and adults with CKD (2-4). Changes in the perception of taste, early satiety signals due to delayed gastric emptying, nausea and vomiting cause insufficient energy and nutrient intake in CKD (3-6). Therefore, children with CKD are significant risk group for protein energy malnutrition (PEM). In an extensive study of 5022 children conducted in North America, it was found that onethird of children with CKD had PEM and this rate was higher in the younger age group (7). The best known cause of PEM in chronic kidney disease is the inadequacy of nutrient storage of the body in response to an increase in nutritional requirements arising from rapid growth (1).

Nutritional status has a great influence on neurocognitive and sexual development of children besides the growth and development rate (8). The growth of all children should therefore be monitored at all stages of CKD and dialysis and nutritional status should be evaluated periodically. Dietary management and manipulation should be used, considering individual dietary habits and cultures.

This study was conducted on children with CKD who were followed at the pediatric nephrology clinic of our center to evaluate their dietary habits, malnutrition status and dietary management practices.

\section{MATERIAL and METHOD}

The study was conducted between November 2016 and May 2017 on 16 patients aged between 2-18 years of age (9 boys, 7 girls) with predialysis CKD and with peritoneal dialysis (PD) who were followed at the University of Health Sciences, Ankara Hematology and Oncology Division of Pediatric Research and Training Hospital Department of Nephrology. Approval of local ethics committee was obtained with the decision no. 2017/060.

Nutritional status of the patients was determined by means of 24-hour dietary recall and subjective global nutritional assessment (SGNA). SGNA is a nutritional assessment tool used collectively and systematically. Subjective global nutritional assessment includes five components of a medical history (weight change, dietary intake, gastrointestinal (Gl) symptoms, functional capacity, metabolic stress) and two components of a brief physical examination (signs of fat loss and muscle wasting, alterations in fluid balance). The results of these components are used to classify patients as "normally nourished," "moderately malnourished" or "severely malnourished" and reflect a reliance on clinical judgment rather than biochemical or other objective markers to categorize nutritional status (9).

Dietary energy and nutrient intake were evaluated by Recommended Dietary Allowance (RDA). Recommended daily intake levels as intercept points [(2/3=67\%) $\pm 33 \%]$ were calculated. Recommended dietary energy and nutrients intake considered as "sufficient" for the average levels of $67-133 \%$, "insufficient" for the avarage levels of $<67 \%$ and as "extreme" for the levels $>133 \%$ (10).

Data were obtained using the face-to-face survey method. The survey included general information, identification of nutritional status, information about the family, 24-hour dietary recall, anthropometric measurements, biochemical findings and SGNA test used for malnutrition scan in children. The food consumption record in our survey was taken using the 24hour diet recall method. Biochemical data include creatinine, uric acid, total protein, albumin, hemoglobin $(\mathrm{Hb})$, hematocrit (Hct), ferritin, iron, iron binding capacity, sodium, potassium, phosphorus, triglyceride, total cholesterol, high density lipoprotein (HDL), low density lipoprotein (LDL), and very low density lipoprotein (VLDL) values. Glomerular filtration rate (GFR) was calculated by Shwartz formula.

Anthropometric measurements were made by researchers using a measuring tape, scale and Holtain skinfold caliper. Body weights of patients was measured using electronic precision scale (with pre-dialysis weight and dry weight for those who receive dialysis treatment), while height measurements were made by a tape measure based on the Frankfort plane. Upper middle arm circumference was measured by a tape measure after marking the middle point between acromial protrusion on the shoulder and olecranon protrusion on the elbow when the arm is bent at 90 degrees from the elbow, triceps skinfold thickness was measured from the marked point with right hand using caliper after holding the skinfold with left hand forefinger and thumb by releasing the arm.

Statistical analysis of the data obtained from the survey was made using statistical package for the social sciences (SPSS) and the food consumption records were evaluated using nutrition information system (BeBIS) computer programs. 


\section{RESULTS}

Of the children with CKD who participated in this study, 56.3\% were female (n:9) and $43.7 \%$ were male (n:7). Median age for girls and boys was 6 and 13 years, respectively.

When anthropometric measurements of children were evaluated; $56.2 \%$ were found to be wasting and $62.5 \%$ were found to be stunting. According to SGNA, 60\% were found to be severely malnourished.

Five of the children (31.2\%) are subjected to dialysis. Classification of the children according to the stage of the chronic kidney disease and the type of dialysis is shown in Table I.

In this study, iron binding capacity, GFR and albumin levels were significantly higher in the predialysis group than in the peritoneal dialysis group $(p<0.05)$. When uric acid, total protein, $\mathrm{Hb}$, Hct, ferritin, iron, sodium, potassium, and phosphor levels were examined, no difference was found between the groups ( $p>0.05$ ) (Table II).

When school attendance was examined, it was found that $50 \%$ of participants left the school, while others continue their education.

$29 \%$ of the patients reported loss of appetite and 12.9\% reported loss of body weight. When they were asked whether they had received nutritional supplements, it was found that $22.2 \%$ of the girls and $14.3 \%$ of the boys were using pediatric enteral nutrition product.

$50 \%$ of children with chronic kidney disease have special nutritional planning, while other $50 \%$ don't. $66.7 \%$ of those who recommended the diet were physicians while $33.3 \%$ were dietitians. Regarding the source of information they used about nutrition of children, $20 \%$ of families stated that they used their own experiences, 20\% applied pediatrician's suggestions and $5 \%$ relied on family physician's suggestions. However, $10 \%$ of patients relied on dietitian's suggestions, $5 \%$ on internet, $\% 10$ on close friends and $5 \%$ TV. $25 \%$ of patients stated that they did not have benefit from any source. $25 \%$ of the patients had a fat consumption restriction in their diets, while $75 \%$ had no fat restriction. The preferences of fat used by families when cooking were rated as 3 point for the first preference, 2 point for the second and 1 point for the last preference. Accordingly, sunflower oil was ranked first with 39 points, olive oil second with 20 points, butter third with 16 points, margarine fourth with 12 points, and hazelnut oil fifth with 1 point.

$68.7 \%$ of the patients consume snack foods before meals. The most preferred snacks in order were ready-made products (biscuits, chocolate, cakes, sweets, pudding) with 29 points, oily foods (popcorn, French fries, chips) with 13 points, fruits with 12 points, soft drinks with 9 points (fruit juice, Nescafe, ice tea, cola), soup, bread, bagels and sandwich with 8 points, salty foods (dried nuts, crackers) with 7 points, oily seeds and olive with 5 points, cheese with 3 points, and fruit yogurt with 2 points.

When we look at the data on children's salt consumption; $31.3 \%$ stated that they consume their foods without salt, $43.7 \%$ prefer less salted, $12.5 \%$ consume normal salty, and $12.5 \%$ consume their foods salty. The percentage of participants who consume juiceless or drained way vegetable meals was $31.3 \%$; $62.5 \%$ said they do not consume in this manner, and $6.2 \%$ said they sometimes consume this way.

In this study, $87.5 \%$ of children with CKD have breakfast and dinner every day and skip the lunch and the percentage of those who eat all three snacks were $37.4 \%$. When it comes to reasons for skipping a meal, it was found that loss of appetite is the most important reason (53.8\%).

Regarding the children's eating problems; 50\% had problems with chewing and swallowing. $43.7 \%$ had a history of improper behavior while eating. $56.3 \%$ of patients stated that they do not like to eat and feel themselves satiated (Table III).

Table I: Stage of the chronic kidney disease and the dialysis methods applied to children.

\begin{tabular}{|c|c|c|c|c|c|c|}
\hline \multirow{2}{*}{ Type of Dialysis } & \multicolumn{2}{|c|}{ Girl } & \multicolumn{2}{|c|}{ Boy } & \multicolumn{2}{|c|}{ Total } \\
\hline & $\mathbf{n}$ & $\%$ & $\mathbf{n}$ & $\%$ & $\mathbf{n}$ & $\%$ \\
\hline Predialysis & 6 & 66.6 & 5 & 71.4 & 11 & 68.8 \\
\hline Total & 9 & 100 & 7 & 100 & 16 & 100 \\
\hline \multicolumn{7}{|l|}{ Type of Peritoneal Dialysis } \\
\hline $\begin{array}{l}\text { Automated peritoneal dialysis } \\
\text { (APD) }\end{array}$ & 1 & 33.3 & - & - & 1 & 20 \\
\hline CAPD and APD & 1 & 33.3 & - & - & 1 & 20 \\
\hline Total & 3 & 100 & 2 & 100 & 5 & 100 \\
\hline
\end{tabular}




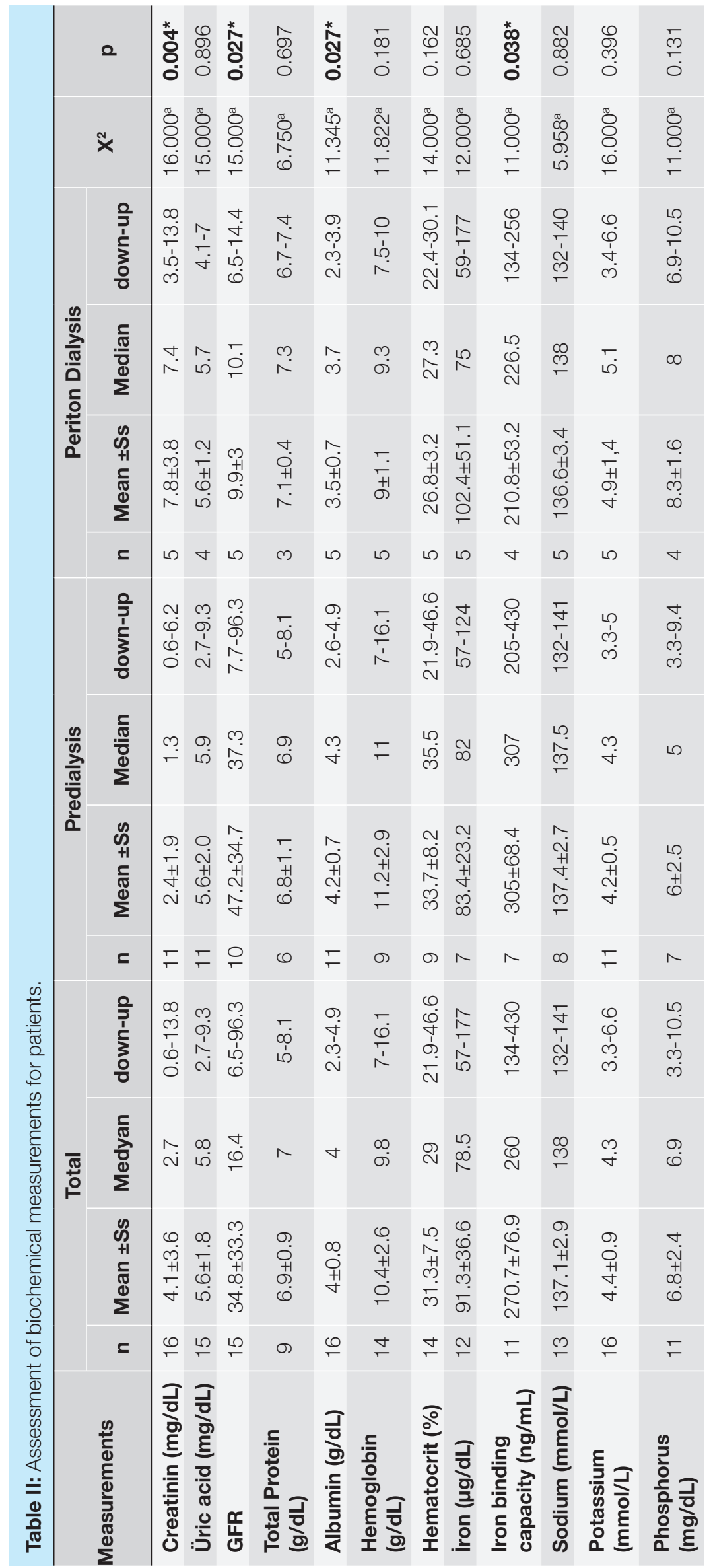


Table III: Determination of children's nutritional problems according to SGNA.

\begin{tabular}{l|c|c|c|c}
\hline SGNA Nutrition & \multicolumn{2}{c}{} & \multicolumn{2}{c}{ No } \\
\hline Nutritional problem & $\mathbf{n}$ & Yes & $\mathbf{n}$ & $\%$ \\
\hline Difficulty in chewing and swallowing the food & 8 & 50 & 8 & 50 \\
\hline Inappropriate behavior during meal & 7 & 43.7 & 9 & 56.3 \\
\hline Dislike of food & 9 & 56.3 & 7 & 43.7 \\
\hline Feeling full & 9 & 56.3 & 7 & 43.7 \\
\hline Food allergy & 1 & 6.3 & 15 & 93.7 \\
\hline
\end{tabular}

Table IV: Determination of nutritional status of children according to SGNA.

\begin{tabular}{l|l|l}
\hline SGNA Nutrition & $\mathbf{n}$ & $\%$ \\
\hline Generally Consumed Food Group & & \\
$\quad$ Cereals & 8 & 50 \\
$\quad$ Vegetable and Fruit & 2 & 12.4 \\
$\quad$ Meat, fish, chicken, other & 1 & 6.3 \\
$\quad$ Milk and milk products & 5 & 31.3 \\
\hline
\end{tabular}

\section{Appetite Status}

\begin{tabular}{|l|l|l|}
\hline Excellent & 4 & 25 \\
\hline Moderate & 8 & 50 \\
\hline Good & 2 & 12.5 \\
\hline Bad & 2 & 12.5 \\
\hline
\end{tabular}

Change in Oral Intake (in the last 15 days)

\begin{tabular}{|l|r|l|}
\hline No & 6 & 37.5 \\
\hline Yes & 10 & 62.5 \\
\hline Increased & 6 & 60 \\
\hline Decreased & 4 & 40 \\
\hline
\end{tabular}

Family Members Who Use Special Diets

\begin{tabular}{l|l|l|}
\hline None & 16 & 100 \\
\hline Present & - & - \\
\hline ildren's State of Adherence to Diet & & \\
\hline No & 8 & 50 \\
\hline Yes & 8 & 50 \\
\hline
\end{tabular}

State of Changing the Nutrients They Eat and Drink

\begin{tabular}{|l|l|l|}
\hline No & 7 & 43.8 \\
\hline Yes & 9 & 56.3 \\
\hline
\end{tabular}

\section{Method of Change}

\begin{tabular}{|l|l|l|}
\hline Salt-free consumption & 6 & 50 \\
\hline Low fat consumption & 2 & 16.8 \\
\hline Not consuming gaseous products & 1 & 8.3 \\
\hline Sugar-free consumption & 1 & 8.3 \\
\hline Not consuming processed food & 1 & 8.3 \\
\hline Supplement reinforcement & 1 & 8.3 \\
\hline
\end{tabular}

When children's nutritional status is examined according to SGNA method, it was found that $50 \%$ of them eat based on the grains, and $25 \%$ were found to be "perfect" and $50 \%$ were 'moderate' in terms of appetite. When we look for apetite change within two weeks, $60 \%$ of children over two weeks had increased oral intake. In all families of children, there were no other individuals who have a special diet. When we look at their CKD specific dietary practices, it was found that 50\% of participants do not follow their diet. 50\% of children who adhere to the diet program consume salt-free diet, while 16.8\% consume less fat diet. The use of additional supplements is $8.3 \%$. The percentage of patients who do not consume processed foods is $8.3 \%$ (Table IV).

According to the food consumption records of children; daily energy intake and carbohydrate intake was found sufficient in $56.3 \%$ and $50 \%$, respectively. And diet protein intake was found to be high in $68.7 \%$ (Table IV).

Breakdown of diets by macronutrients is given in Table $\mathrm{V}$.

The average daily energy intake of children is $1564.3 \pm 982.4$ kcal. $45.6 \pm 9.0 \%$ of the intake energy is from carbohydrate $(\mathrm{CHO}), 12.4 \pm 4.1 \%$ from the protein and $41.9 \pm 7.0 \%$ from the fat. $21.0 \pm 14.6 \mathrm{~g}$ of dietary fat comes from saturated fats, $18.1 \pm 19.0 \%$ from polyunsaturated fats and $20.4 \pm 17.2 \%$ from monounsaturated fats. Daily cholesterol intake in diet is $199.0 \pm 144.7 \mathrm{mg}$.

It was found that children with chronic kidney disease had inadequate intake of dietary fiber, calcium and magnesium, and consumed salt more than needed (Table VI).

\section{DISCUSSION}

In the early stages of CKD, strict nutritional intervention can prevent more serious complications of nutrition in the disease process. There is a great need for methods that assessment of nutritional status extensively in all stages of CKD and in dialysis treatment. However, there are limited clinical trials for assessing nutritional status of children with CKD (11). For this reason, the study was conducted to evaluate dietary habits, malnutrition status and dietary management of 16 (9 girls, 7 boys) patients, including 11 with predialysis and 5 PD treatment. 


\begin{tabular}{|c|c|c|c|c|c|c|c|c|c|c|c|c|c|c|}
\hline & 0 & $\mid \begin{array}{ll} & 5 \\
0 & 5 \\
0 & 0\end{array}$ & 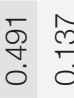 & $\frac{\Gamma}{i}$ & $\begin{array}{l}\hat{f} \\
\stackrel{f}{0} \\
0\end{array}$ & $\begin{array}{l}\frac{10}{0} \\
0 \\
0\end{array}$ & $\frac{\stackrel{\llcorner}{N}}{\stackrel{\circ}{\circ}}$ & $\begin{array}{l}\infty \\
\stackrel{\leftrightarrow}{O} \\
0\end{array}$ & $\begin{array}{l}0 \\
\stackrel{m}{0} \\
\frac{0}{0}\end{array}$ & $\begin{array}{l}\bar{\sigma} \\
\stackrel{0}{0} \\
0\end{array}$ & $\frac{\stackrel{\sim}{N}}{\stackrel{0}{\circ}}$ & $\begin{array}{l}\stackrel{+}{N} \\
\infty \\
0 \\
0\end{array}$ & $\begin{array}{l}\infty \\
\infty \\
\\
0\end{array}$ & $\begin{array}{l}\bar{\sigma} \\
\text { న. } \\
0\end{array}$ \\
\hline & $\tilde{x}$ & 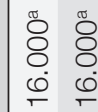 & 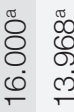 & 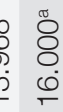 & $\begin{array}{l}\tilde{0} \\
00 \\
0 \\
\infty \\
\infty \\
\Gamma\end{array}$ & $\begin{array}{l}8 \\
8 \\
0 \\
0\end{array}$ & $\begin{array}{l}8 \\
8 \\
0 \\
0\end{array}$ & $\begin{array}{l}8 \\
8 \\
0 \\
0\end{array}$ & 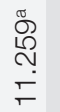 & $\begin{array}{l}8 \\
8 \\
0 \\
0\end{array}$ & $\begin{array}{l}8 \\
8 \\
0 \\
0\end{array}$ & $\begin{array}{l}8 \\
8 \\
0 \\
0 \\
\end{array}$ & $\begin{array}{l}8 \\
8 \\
0 \\
\oplus\end{array}$ & $\begin{array}{l}8 \\
8 \\
0 \\
0 \\
10\end{array}$ \\
\hline & 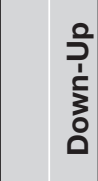 & 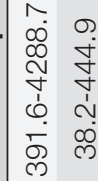 & 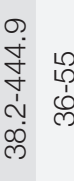 & $\begin{array}{ll}0 \\
0 \\
b \\
b \\
b \\
0 \\
0\end{array}$ & 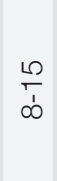 & $\begin{array}{l}\infty \\
0 \\
0 \\
0 \\
+ \\
+ \\
+\end{array}$ & $\begin{array}{l}0 \\
\infty \\
\infty \\
⿱ \\
\stackrel{1}{N}\end{array}$ & $\begin{array}{l}\hat{\infty} \\
\stackrel{\infty}{N} \\
\dot{\omega} \\
\stackrel{\sim}{\sim} \\
\end{array}$ & $\begin{array}{l}\text { N̂ } \\
0 \\
\varnothing\end{array}$ & $\begin{array}{l}0 \\
0 \\
0 \\
0 \\
0 \\
\dot{+}\end{array}$ & $\begin{array}{l}\infty \\
\stackrel{+}{+} \\
\stackrel{1}{\perp}\end{array}$ & $\begin{array}{l}0 \\
\phi \infty \\
0 \\
\dot{\omega} \\
\infty\end{array}$ & 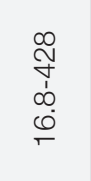 & $\begin{array}{l}\stackrel{m}{P} \\
\stackrel{P}{+} \\
+\end{array}$ \\
\hline & $\begin{array}{l}\frac{\tilde{\sigma}}{\bar{z}} \\
\frac{0}{\Sigma} \\
\Sigma\end{array}$ & 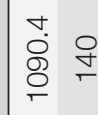 & $\stackrel{\circ}{+}$ & 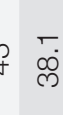 & $F$ & $\begin{array}{l}\stackrel{0}{\dot{\theta}} \\
\stackrel{\sim}{N}\end{array}$ & $\begin{array}{l}\stackrel{\sim}{\sim} \\
\leftarrow\end{array}$ & $\begin{array}{l}\stackrel{\leftrightarrow}{\sim} \\
\stackrel{f}{*}\end{array}$ & g & $\begin{array}{l}\stackrel{N}{0} \\
\stackrel{0}{\leftarrow}\end{array}$ & 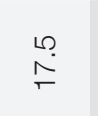 & $\stackrel{\infty}{\infty}$ & 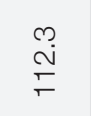 & 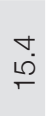 \\
\hline & $\begin{array}{l}\text { ठ } \\
+1 \\
+ \\
\bar{\Xi} \\
\sum \\
\Sigma\end{array}$ & 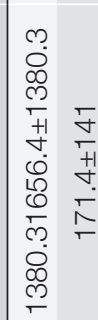 & 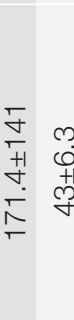 & 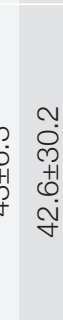 & 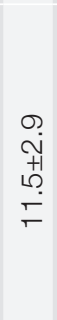 & 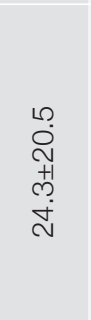 & $\begin{array}{l}\stackrel{\sim}{+} \\
\stackrel{+}{+} \\
+ \\
+ \\
\infty \\
\leftarrow\end{array}$ & $\begin{array}{l}\infty \\
\infty \\
\infty \\
+1 \\
\infty \\
0 \\
\infty\end{array}$ & 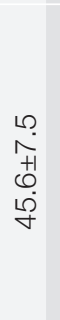 & 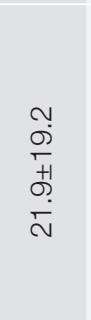 & 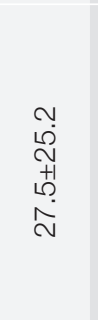 & $\begin{array}{l}\hat{N} \\
\stackrel{N}{ } \\
+ \\
\stackrel{N}{+} \\
\dot{N}\end{array}$ & 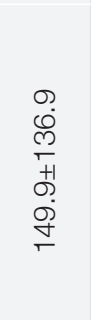 & 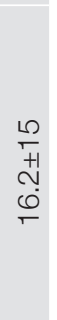 \\
\hline & c & $\wedge \wedge$ & $\wedge \wedge$ & -1 & $\wedge$ & $\wedge$ & $\wedge$ & $\wedge$ & $\wedge$ & $\wedge$ & $\wedge$ & $\wedge$ & $\wedge$ & $\wedge$ \\
\hline & 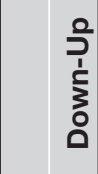 & 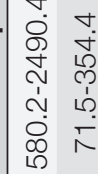 & 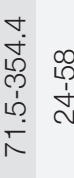 & 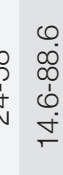 & 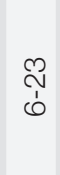 & $\begin{array}{l}+ \\
\infty \\
0 \\
0 \\
0 \\
0 \\
0\end{array}$ & 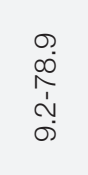 & 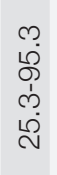 & $\begin{array}{l}0 \\
0 \\
0 \\
0\end{array}$ & $\begin{array}{l}\text { ते. } \\
\text { ले } \\
\text { ले }\end{array}$ & 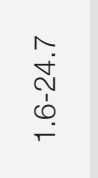 & 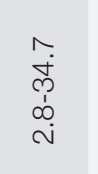 & 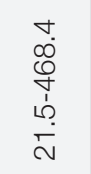 & $\begin{array}{l}\bar{i} \\
\text { Dे } \\
\omega \\
0 \\
0\end{array}$ \\
\hline & 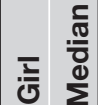 & 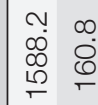 & 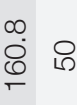 & $\stackrel{ナ}{\stackrel{\forall}{\forall}}$ & $\stackrel{\simeq}{\simeq}$ & $\begin{array}{l}\infty \\
\stackrel{\sim}{\sim}\end{array}$ & $\stackrel{+}{\stackrel{+}{\sim}}$ & 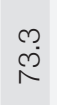 & $\stackrel{\infty}{\infty}$ & $\hat{\bar{c}}$ & $\begin{array}{l}\stackrel{N}{\infty} \\
\infty\end{array}$ & $\begin{array}{l}\stackrel{\leftrightarrow}{\sim} \\
\stackrel{\sim}{\sim}\end{array}$ & $\underset{\infty}{\infty}$ & $\simeq$ \\
\hline $\begin{array}{l}\stackrel{2}{r} \\
0 \\
c\end{array}$ & $\begin{array}{l}\text { क } \\
+1 \\
+1 \\
\bar{\varpi} \\
\Sigma\end{array}$ & 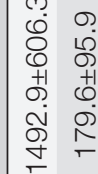 & 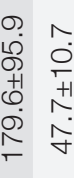 & 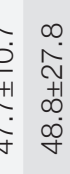 & $\begin{array}{l}0 \\
\dot{O} \\
+ \\
+ \\
N \\
\stackrel{D}{+}\end{array}$ & $\begin{array}{l}\stackrel{M}{\mathcal{N}} \\
\stackrel{+}{+1} \\
\stackrel{0}{+} \\
\stackrel{+}{\leftarrow}\end{array}$ & 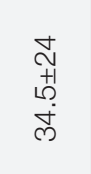 & $\begin{array}{l}\infty \\
\stackrel{3}{ } \\
\stackrel{1}{+1} \\
\infty \\
\tilde{0}\end{array}$ & 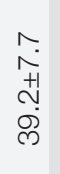 & $\begin{array}{l}\stackrel{\infty}{-} \\
\stackrel{+}{+1} \\
\stackrel{+}{+} \\
\stackrel{N}{*}\end{array}$ & $\begin{array}{l}\infty \\
+1 \\
\infty \\
0 \\
0 \\
0\end{array}$ & $\begin{array}{l}\stackrel{0}{0} \\
0 \\
++1 \\
\stackrel{+}{0} \\
\sim \\
\sim\end{array}$ & $\begin{array}{l}0 \\
\dot{0} \\
0 \\
+ \\
+ \\
N \\
N \\
\widetilde{N}\end{array}$ & 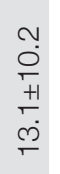 \\
\hline & c & a 0 & の の & 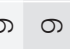 & $\sigma$ & o & $\sigma$ & $\infty$ & $\sigma$ & $\sigma$ & $\sigma$ & $\sigma$ & $\sigma$ & $\sigma$ \\
\hline 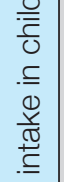 & \begin{tabular}{l}
0 \\
\multirow{1}{1}{} \\
3 \\
0 \\
0
\end{tabular} & 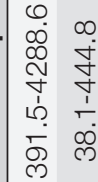 & 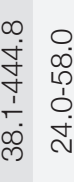 & 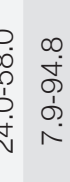 & $\begin{array}{l}0 \\
\grave{N} \\
i \\
0 \\
\dot{0}\end{array}$ & 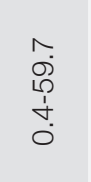 & $\begin{array}{l}0 \\
\infty \\
\infty \\
\vdots \\
o \\
\stackrel{N}{j}\end{array}$ & 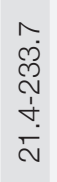 & $\begin{array}{l}0 \\
\text { ભ } \\
0 \\
0 \\
0 \\
0 \\
0\end{array}$ & $\begin{array}{l}0 \\
0 \\
0 \\
0 \\
0 \\
\infty\end{array}$ & 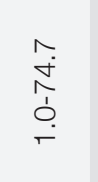 & $\begin{array}{l}0 \\
\varnothing 0 \\
0 \\
0 \\
0 \\
i\end{array}$ & 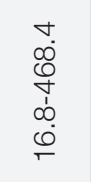 & $\begin{array}{l}m \\
\dddot{m} \\
\stackrel{5}{+} \\
\omega \\
0\end{array}$ \\
\hline 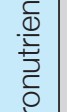 & 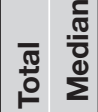 & 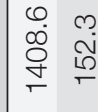 & 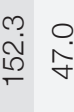 & 我 & ㅇ. & 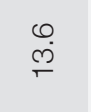 & 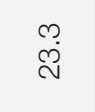 & 广্ं & $\stackrel{\circ}{\stackrel{\leftrightarrow}{f}}$ & $\begin{array}{l}\stackrel{0}{\circ} \\
\stackrel{\Gamma}{\Gamma}\end{array}$ & $\begin{array}{l}0 \\
\stackrel{0}{\llcorner}\end{array}$ & $\begin{array}{l}\stackrel{\rho}{\infty} \\
\stackrel{\Gamma}{\leftarrow}\end{array}$ & $\begin{array}{l}\infty \\
\stackrel{\infty}{N} \\
\Gamma\end{array}$ & $\underset{\sim}{\stackrel{N}{\sim}}$ \\
\hline 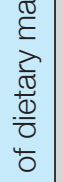 & 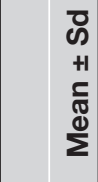 & 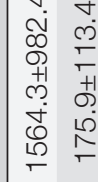 & 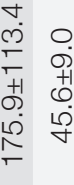 & 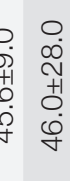 & $\begin{array}{l}- \\
\dot{+} \\
+ \\
\dot{+} \\
\stackrel{+}{\sigma}\end{array}$ & $\begin{array}{l}0 \\
\dot{0} \\
++1 \\
0 \\
0 \\
\infty \\
\leftarrow\end{array}$ & \begin{tabular}{l}
$\stackrel{\infty}{\sim}$ \\
$\stackrel{\sim}{N}$ \\
+ \\
\multirow{\sim}{*}{}
\end{tabular} & $\begin{array}{l}\infty \\
\infty \\
\infty \\
1 \\
\infty \\
\infty \\
\\
\dot{n}\end{array}$ & $\begin{array}{l}\circ \\
\infty \\
0 \\
+1 \\
+ \\
+ \\
+\end{array}$ & $\begin{array}{l}0 \\
\dot{+} \\
\Gamma+1 \\
0 \\
\dot{⿰}\end{array}$ & $\begin{array}{l}\stackrel{0}{\circ} \\
\stackrel{+}{+1} \\
\stackrel{+1}{\circ} \\
\stackrel{\infty}{\leftarrow}\end{array}$ & $\begin{array}{l}\stackrel{N}{N} \\
\stackrel{+}{+} \\
+1 \\
\stackrel{N}{+}\end{array}$ & 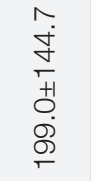 & 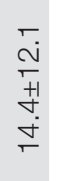 \\
\hline . & c & $\stackrel{\varphi}{\varphi} \stackrel{\varphi}{2}$ & $\stackrel{\varphi}{\circ} \stackrel{0}{\square}$ & 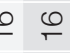 & $\stackrel{\varphi}{\leftarrow}$ & $\stackrel{\varphi}{\leftarrow}$ & $\tau$ & $\stackrel{\varphi}{\leftarrow}$ & $\stackrel{\varphi}{\leftarrow}$ & $\stackrel{6}{\circ}$ & $\stackrel{\varphi}{\circ}$ & $\stackrel{\varphi}{\sim}$ & $\underset{\sim}{\varphi}$ & $\underset{\tau}{\varphi}$ \\
\hline $\begin{array}{l}\frac{\overrightarrow{0}}{0} \\
\text { 山े } \\
\frac{0}{0} \\
\frac{0}{0}\end{array}$ & 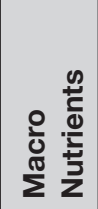 & 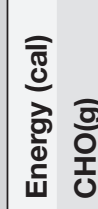 & 흠 $\frac{0}{\circ}$ & 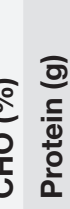 & 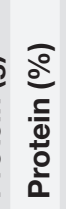 & 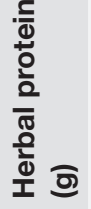 & 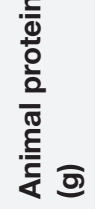 & $\begin{array}{l}\text { 호 } \\
\text { 휴 }\end{array}$ & $\frac{\varlimsup}{\grave{\vdots}}$ & 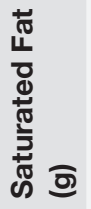 & 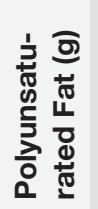 & 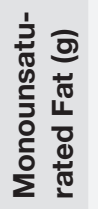 & $\begin{array}{l}\overline{0} \\
\frac{0}{\Phi} \\
\bar{\phi} \\
\frac{\Phi}{0} \\
\frac{0}{0} \text { 이 }\end{array}$ & $\begin{array}{l}\text { 후 } \\
\text { 히 } \\
\text { 은 }\end{array}$ \\
\hline
\end{tabular}


Protein energy malnutrition is the most important risk factor in children with chronic kidney disease. Nutrition is the most important factor for children. Because the growth retardation encountered in this period can not be recovered entirely. Therefore, nutritional care plans in children have focused on continuation of optimal nutritional status in all of the spectrums of CKD to avoid uremic toxicity, metabolic abnormalities and malnutrition (8). In this study, $62.5 \%$ of the children had severe malnutrition according to the SGNA method. In the study conducted by Gupta et al. (12), moderate to severe malnutrition was found in $60 \%$ of the children. As in this study, no obese or fat patients were found.

The short stature in children is the long-term sequelae of endstage renal failure. While adversely affecting the quality of life of children psychologically, it makes social integration difficult in their transition to adulthood (13). In a study conducted in the USA on 799 children with CKD, 12\% were found to be seriously stunted (14). In this study, $62.5 \%$ of the children were found to be short by their age. The growth of children who receive renal replacement therapy falls behind despite modern hormonal therapy methods and nutritional support (15). Inadequate growth in children with CKD is associated with an increased risk of admission and death, and risk of death increases by $14 \%$ with each unit of decrease in each unit height SDS. The risk of mortality doubles when the stature SDS is 2.6 (16).

When school attendance status of the children participating in the study was examined; it was found that $50 \%$ of children continue their education, while 50\% left the school. According to the SGNA method, the reasons for non-attendance in school were mental retardation with $11.1 \%$, irregularity of blood pressure with $11.1 \%$, risk of infection with $11.1 \%$, severe illness with $11.1 \%$, and studying distance education with $33.3 \%$. It was shown in a related study that health-associated quality of life in children with CKD who underwent long-term dialysis was lower than in children with chronic non-cancer diseases (17). Anemia, HT, microvascular diseases, malnutrition, and neurocognitive development of seizures negatively affect academic achievement especially in dialysis patients $(18,19)$. Also, it was reported that they could not continue their education, have difficulties in adaptation to adulthood, and do not sustain their independent life (20).

Table VI: Daily dietary nutrients sufficiency of the children with CKD.

\begin{tabular}{|c|c|c|c|c|c|c|}
\hline \multirow{2}{*}{ Nutrient } & \multicolumn{2}{|c|}{ Insufficient } & \multicolumn{2}{|c|}{ Sufficient } & \multicolumn{2}{|c|}{ Excessive } \\
\hline & $\mathbf{n}$ & $\%$ & $\mathbf{n}$ & $\%$ & $\mathbf{n}$ & $\%$ \\
\hline Energy & 5 & 31.3 & 9 & 56.3 & 2 & 12.4 \\
\hline Carbohydrate & 4 & 25 & 8 & 50 & 4 & 25 \\
\hline Protein & 1 & 6.3 & 4 & 25 & 11 & 68.7 \\
\hline Herbal Protein & 4 & 25 & 2 & 12,5 & 10 & 62,5 \\
\hline Herbal Protein & 3 & 18.7 & 4 & 25 & 9 & 56.3 \\
\hline Fat & - & - & 7 & 43.8 & 9 & 56.2 \\
\hline Fiber & 10 & 62.5 & 5 & 31.2 & 1 & 6.3 \\
\hline Vitamin A & 5 & 31.2 & 5 & 31.2 & 6 & 37.6 \\
\hline Vitamin E & 7 & 43.7 & 1 & 6.3 & 8 & 50 \\
\hline Vitamin K & 2 & 12.5 & 3 & 18.8 & 11 & 68.7 \\
\hline Vitamin C & 3 & 18,7 & 7 & 43,7 & 6 & 37,6 \\
\hline Vitamin B1 & 7 & 43.6 & 6 & 37.6 & 3 & 18.8 \\
\hline Vitamin B2 & 4 & 25 & 5 & 31.3 & 7 & 43.7 \\
\hline Vitamin B3 & 8 & 50 & 6 & 37.7 & 2 & 6.3 \\
\hline Vitamin B5 & 5 & 31.3 & 7 & 43.7 & 4 & 25 \\
\hline Vitamin B6 & 4 & 25 & 7 & 43.7 & 5 & 31.3 \\
\hline Vitamin B9 & 12 & 75 & 3 & 18.7 & 1 & 6.3 \\
\hline Vitamin B12 & 3 & 18.7 & 5 & 31.3 & 8 & 50 \\
\hline Sodium & - & - & 2 & 12.5 & 14 & 87.5 \\
\hline Potassium & 6 & 37.6 & 5 & 31.2 & 5 & 31.2 \\
\hline Calcium & 10 & 62.5 & 6 & 37.5 & - & - \\
\hline Magnesium & 9 & 56.3 & 4 & 25 & 3 & 18.7 \\
\hline Phosphorus & 2 & 12.5 & 8 & 50 & 6 & 37.5 \\
\hline Iron & 6 & 37.5 & 8 & 50 & 2 & 12.5 \\
\hline Zinc & 4 & 25 & 9 & 56.3 & 3 & 18.7 \\
\hline
\end{tabular}


In this study, iron binding capacity, GFR and blood albumin levels were significantly higher in the predialysis group than in the PD group $(p<0.05)$ (Table II). The prevalence of anemia increases as the stage progresses in CKD. The most important reason for the anemia is the absence of erythropoietin use, iron deficiency, malnutrition and presence of parasitic infections (12) because of the cost of treatment in the final stages. In their study, Gupta et al. (12) found that prevalence of anemia increased from $45.7 \%$ to $100 \%$ in the last stage CKD as the stage progressed. Similarly, as the stage progressed, the prevalence of hypoalbuminemia increased and GFH decreased.

In this study, blood uric acid, total protein, Hb, Hct, ferritin, sodium, potassium and phosphor levels were found similar in predialysis group and dialysis group ( $p>0.05)$. Apostoloue et al. (21) found that these values were lower in dialysis-treated children than in children with stage III-IV CKD.

Infants and children with chronic kidney disease are at a higher risk of mortality and morbidity as they are in the stage of growth and development (22). A detailed nutritional counseling is needed to assess individual nutritional status, identify the presence of malnutrition, develop a patient-specific diet, provide oral and parenteral nutritional support, and replace protein deficiencies (23). In this study, $50 \%$ of children with CKD have a special dietary plan while others do not. $66.7 \%$ received diet-related recommendations from physicians, $33.3 \%$ from dietitian and only $10 \%$ of the families stated that they benefited from the dietitian as a source about the nutrition of child. However, according to the CKD nutrition guidelines, it is recommended that children be regularly assessed for their nutritional status. Dietary management should be performed by dieticians specialized in pediatric and renal nutrition (taking into account the child's age, growth and development status, nutritional preferences, cultural differences, and psychosocial state (22).

Dietary habits and appetite status of children with chronic kidney disease are earliest indicators of nutritional status. A study conducted in Korea found that dietary habits and appetite status in children with CKD are associated with a decrease in kidney functions. In addition, the order of meals, frequency of main and break meals, having regular breakfast, excessive appetite and loss of appetite are recognized as determinants of diet quality (1). In this study, $87.5 \%$ of children with CKD have breakfast and dinner every day and skip the lunch. 37.5\% have all three snacks every day. When it comes to reasons for skipping a meal, 53.8\% stated that they do not eat because of loss of appetite. When children are evaluated based on their eating habits, it was found that $50 \%$ had chewing and swallowing problems and $56.3 \%$ felt full (Table III). Feeling of fullness is due to reabsorption of dialysate glucose and intraabdominal filling in PD treatment (24). Also, it was found in this study that $68.7 \%$ of children with CKD consumed pre-meal snacks, which is believed to suppress their appetite during the main meal.
A study conducted on the effect of nutritional counseling on diet quality in chronic kidney disease reported that a training consisting of only food and nutrient restrictions does not improve diet quality (25). Similarly, $50 \%$ of children do not comply with CKD-specific dietary recommendations in this study. It was found that of the children who stated that they adhere to their diets, $50 \%$ eat salt-free, $16.8 \%$ restrict diet fat, $50 \%$ eat based on cereals, $31.3 \%$ eat based on milk and milk products, and $12.4 \%$ generally eat vegetables and fruits. Only 8.3\% use additional supplements, while $8.3 \%$ do not consume ready-to-eat nutrients (Table IV). In a study conducted on diet quality in CKD, it was found that the consumption of milk, vegetables and fruit by the patients was low but they consumed more meat, eggs and legumes (25).

Nutrition guidelines for children with chronic kidney disease of 2008 stated that daily energy needs should be up to $100 \%$ of the recommended amount for age. In this study, energy intake and carbohydrate intake was found sufficient in $56.3 \%$ and $50 \%$ of children, respectively. Protein and fat intake is above the recommendation. The percentage of dietary energy from fat is $41.9 \pm 8.0$ (Table V). The guide suggests that the dietary protein should be $100 \%$ of the recommended based on ideal body weight with sufficient energy intake and losses should be supplemented with dialysate (21). In the study conducted by Gupta et al. (12), diet protein and dietary fat was also found above the recommended values. Mineral bone disease is a common condition in children with CKD. In this study, calcium and magnesium intake in children with CKD is insufficient and salt intake is higher than the recommended (Table VI).

\section{CONCLUSION}

As a result, children with CKD who were included in this study have irregular eating habits and their dietary management is very poor. Nutritional education, attitudes and behaviors of these children and their families are inadequate. For this reason, it is believed that a diet based on individual nutrition rather than a nutrient-based dietary approach consisting of the restrictions is more suitable for children with CKD. A more liberal and individual dietary management consisting of healthy nutritional choices that increase the quality of diet considering individual nutritional habits will increase the success of multidisciplinary team in the treatment.

The dietician must evaluate the nutritional status and use a scientific language when providing training in a way that the patient and his family can understand. Diet theraphy should be planned and followed regularly, and dietary manipulation should be provided according to findings. The increase in quality of life in CKD is closely related to the increase in quality of nutritional counseling service.

At this stage, there is a need for legal arrangements related to increasing the number of dieticians who are specialized in renal 
pediatric nutrition and can work permanently in the pediatric nephrology team.

\section{ACKNOWLEDGEMENT}

Thank you for your help, collecting the data in this study that $4^{\text {th }}$ class students (Arzu ÜSTÜN and Ayşe Selda ERARSLAN) from Ankara University, Department of Nutrition and Dietetics

\section{REFERENCES}

1. Kim H, Lim H, Choue R. Compromised diet is associated with decreased renal function in children with chronic kidney diesase. Clin Nutr Res 2014; 3: 142-9.

2. Betts PR, Magrath $G$. Growth pattern and dietary intake of children with chronic renal insufficiency. Br Med J 1974;2:189-93.

3. Norman LJ, Coleman JE, Macdonald IA, Tomsett AM, Watson AR. Nutrition and growth in relation to severity of renal disease in children. Pediatric nephrol 2000;15:259-65.

4. Rees L, Jones $\mathrm{H}$. Nutritional management and growth in children with chronic kidney disease. Pediatric Nephrol 2013;28:527-36.

5. Armstrong JE, Laing DG, Wilkes FJ, Kainer G. Smell and taste function in children with chronic kidney disease. Pediatr Nephrol 2010; 25:1497-1504.

6. Rees L, Rigden SP, Ward GM. Chronic renal failure and growth. Arch Dis Child 1989;64:573-7.

7. Mahan JD, Warady BA; Consensus Committee. Assessment and treatmentof short stature in pediatric patients with chronic kidney disease: A consensus statement. Pediatr Nephrol 2006;21:91730.

8. Battelino N, Rus R, Novljan G. Nutritional requirements in children with chronic kidney disease. Clin Nutr ESPEN 2016;14:42-6.

9. Secker DJ, Jeejeebhoy KN. Subjective global nutrition assessment for children. Am J Clin Nutr 2007;85:1083-9.

10. NAP. National Academy Press. Nutrient Adeqency-Asessment Using Food Consumption Surveys. Washington D.C: 1986:14.

11. Graf L, Candelaria S, Doyle M, Kaskel F. Nutrition assessment and hormonal influences on body composition in children with chronic kidney disease. Adv Chronic Kidney Dis 2007;14:215-23.
12. Gupta A, Mantan M, Sethi M. Nutritional assessment in children with chronic kidney disease. Saudi J Kidney Dis Transpl 2016;27:733-9.

13. Rosenkranz J, Reichwald-Klugger E, Oh J, Turzer M, Mehls O, Schaefer F. Psychosocial rehabilitation and satisfaction with life in adults with childhood-onset of end-stage renal disease. Pediatr Nephrol 2005;20:1288-94.

14. Rodig NM, McDermott KC, Schneider MF, Hotchkiss HM, Yadin $\mathrm{O}$, Seikaly MG, et al. Growth in children with chronic kidney disease: A report from the chronic kidney disease in children study. Pediatr Nephrol 2014;29:1987-95.

15. Stanczyk M, Miklaszewska M, Zachwieja K, Wiercin' SR, Stankiewicz R, Firszt AA, et al. Growth and nutritional status in children with chronic kidney disease on maintenance dialysis in Poland. Adv Med Sci 2016;61:46-51.

16. Fischbach M, Fothergill H, Seuge L, Zaloszyc A. Dialysis strategies to Improve growth in children with chronic kidney disease. J Ren Nutr 2011; 21:43-6.

17. Goldstein SL, Graham N, Burwinkle T, Warady B, Farrah R, Varni JW. Health-related quality of life in pediatric patients with ESRD. Pediatr Nephrol 2006;21:846-50.

18. Johnson RJ, Warady BA. Long-term neurocognitive outcomes of patients with end-stage renal disease during infancy. Pediatr Nephrol 2013;28:1283-91.

19. Seliger SL, Weiner DE. Cognitive impairment in dialysis patients: Focus on the blood vessels? Am J Kidney Dis 2013;61:187-90.

20. Neul SK, Minard CG, Currier H, Goldstein SL. Health related quality of life functioning over a 2-year period in children with end-stage renal disease. Pediatr Nephrol 2013;28:285-93.

21. Apostolou A, Printza N, Karagiozoglou-Lampoudi T, Dotis J, Papachristou F. Nutrition assessment of children with advanced stages of chronic kidney disease-A single center study. Hipokratia 2014;18:212-6.

22. KDOQI work group. KDOQI clinical practice guideline for nutrition in children with CKD: 2008 update. Executive summary. Am J Kidney Dis 2009;53:11-104.

23. Druml W, Cano N, Teplan V. Nutritional support in renal disease. In: Sobotka L, (ed). Basics in Clinical Nutrition. 4th ed. Prague: House Galen, 2011:473-85.

24. Warady BA, Neu AM, Schaefer F. Optimal care of the infant, child, and adolescent on dialysis: 2014 update. Am J Kidney Dis 2014;64:128-42.

25. Fernandes AS, Ramos Cl, Nerbass FB, Cuppari L. Diet quality of chronic kidney disease patients and the impact of nutritional counseling. J Ren Nutr 2017;X:1-8. 